\title{
Difficulty in moving around in a wheelchair as a presenting symptom of left atrial myxoma in a chronic paraplegic patient: a case report
}

\author{
A Kovindha and W Saeng-Xuto
}

\section{Study design: Case report.}

Objectives: To present and discuss a case of a chronic paraplegic patient who presented with difficulty in moving around in a wheelchair.

Setting: Rehabilitation Ward, Maharaj Hospital, Faculty of Medicine, Chiang Mai University, Thailand.

Case report: A chronic complete paraplegic T4 man complained of difficulty in moving around in a wheelchair. He had experienced this difficulty for a week. He also complained of pain in the finger pads, and blackening had occurred in his little toes. Buerger's disease was suspected, but Doppler ultrasound showed no evidence of arterial occlusion. During admission, his vision suddenly dropped and magnetic resonance imaging revealed an ischemic stroke in the posterior cerebral artery. Erythematous lesions in all toe pads and soles were actually embolic signs but painless due to his paraplegia. Echocardiography showed a large left atrial myxoma. Six weeks after removal of the left atrial myxoma, he was discharged when he could propel his wheelchair more than $100 \mathrm{~m}$ without difficulty or pain.

Conclusion: Experiencing difficulty in moving around in a wheelchair in conjunction with painful finger pads can be presenting symptoms of a rare cardiac lesion-left atrial myxoma with arterial embolism. After surgical excision of the tumour, the arterial embolic symptoms and signs subsided and the patient regained basic wheelchair mobility skills within 6 weeks.

Spinal Cord (2014) 52, S3-S4; doi:10.1038/sc.2014.78

\section{INTRODUCTION}

Generally, the main clinical presentations of left atrial myxoma are tachyarrhythmias, dyspnea, congestive heart failure, chest pain and syncope followed by neurological and systemic embolic events. ${ }^{1,2}$ However, here we present the case of a chronic paraplegic man with left atrial myxoma and arterial embolism. The diagnosis was delayed due to the uncommon presentation of limited wheelchair mobility with painful finger pads.

\section{CASE REPORT}

A 36-year-old man with complete paraplegic T4 for 16 years complained of a week of pain in all finger pads that markedly limited his ability in moving around in a wheelchair. At the same time, he felt dizzy and noticed dark spots on his little toes. When asked about smoking, he said that he had smoked one cheroot almost every day for 20 years. Physical examination revealed a blood pressure of 96/69, pulse rate of $105 / \mathrm{min}$, normal muscle power of the upper extremities, and erythema and very tender finger pads when touched. He was then admitted with a provisional diagnosis of thromboangiitis obliterans. ECG 12 leads showed normal axis, inverted T in lead II, III, aVF, and a heart rate of 83 beats $\mathrm{min}^{-1}$. Chest $\mathrm{X}$-ray revealed hyperaeration with no cardiomegaly. Doppler ultrasound showed no evidence of arterial occlusion.

A week after admission, from reporting normal vision, the visual acuity of the patient dropped abruptly to $6 / 18$ on the right and 6/24 on the left eye. Ischemic stroke was suspected. Magnetic resonance imaging showed sub-acute ischemic stroke of the posterior cerebral artery with hemorrhagic transformation. Embolism was the most likely cause. We then realized that the skin lesions on all finger and toe pads, and also on the soles of both feet were embolic signs (Figure 1). Echocardiography revealed a large left arterial tumour.

Two days later open-heart surgery with total excision of the tumour was successfully completed. Levophed $0.04 \mathrm{mg} \mathrm{h}^{-1}$ intravenous drip for the first 3 days and aspirin $300 \mathrm{mg}$ daily long-term were prescribed. A week post procedure, when vital signs were stable, he was transferred to the rehabilitation ward. Physical activities were initially limited during the first 2 weeks. Then he was allowed to propel his wheelchair gradually but transferring from bed to wheelchair was assisted. Six weeks post procedure, he was discharged when his hands were pain-free, signs of embolism subsided, and he was able to move around over distances greater than $100 \mathrm{~m}$ and to do samelevel transfer without pain.

When retrospectively reviewing his medical record, 4 months prior to admission, carpal tunnel syndrome was suspected due to his complaint of numbness in both hands but median nerve conduction study was normal.

\section{DISCUSSION}

Previously there had been only one reported case of a chronic paraplegic man complaining of chest oppression due to a large left 


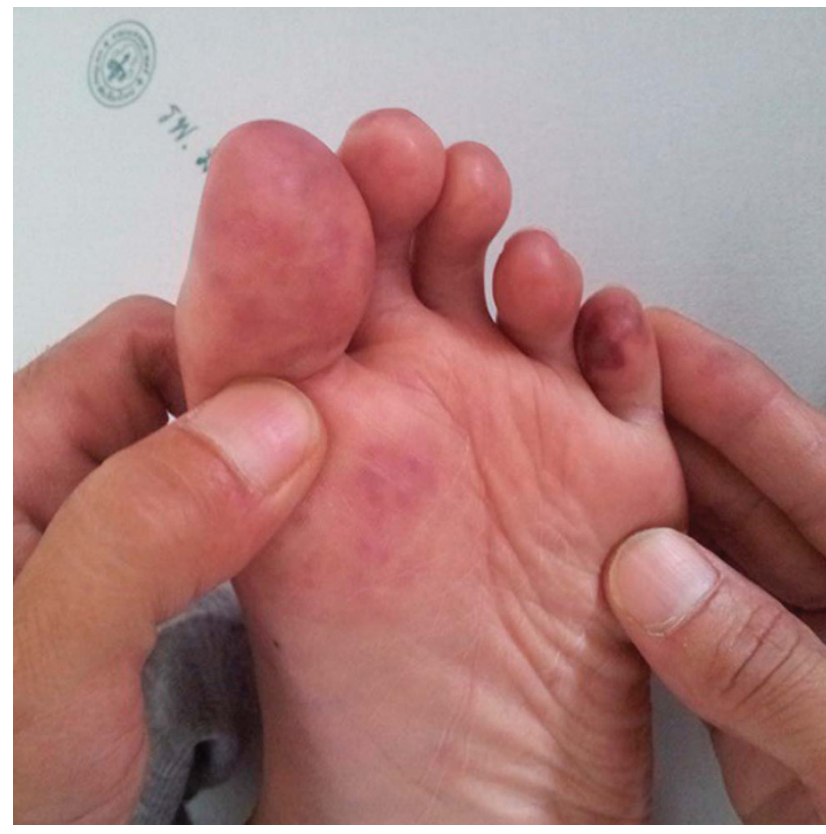

Figure 1 Erythematous skin lesions-Osler's nodes on toe pads and sole.

atrial myxoma. ${ }^{3}$ This is the second documented case of a chronic paraplegic patient who was diagnosed with left atrial myxoma. He firstly presented with numbness in both hands, leading us to a mistaken provisional diagnosis of carpal tunnel syndrome. This condition is prevalent among chronic paraplegic wheelchair users.
When the left atrial myxoma caused arterial emboli to distal parts of all limbs, and painful finger pads markedly limited his ability to move around in a wheelchair, we were further misled to a second provisional diagnosis of thromboangiitis obliterans, which is common among Asian people who smoke or use tobacco. It was not until the patient developed an ischemic stroke that embolism was suspected.

Retrospectively, this patient had embolic signs, that is, Osler's nodes-painful erythematous swelling on finger and toe pads and soles of the feet, but these were painless due to paraplegia. Although such skin lesions are pathognomonic for infective endocarditis, Osler's nodes could be manifestations of distal cutaneous vascular insufficiency from emboli from any sources. ${ }^{4}$

In conclusion, this patient presented with an uncommon complaint of left atrial myxoma-difficulty propelling a wheelchair due to pain in the finger pads, which was actually caused by arterial embolism. The diagnosis of left atrial myxoma was delayed until an ischemic stroke occurred. After removal of the tumour, the patient resumed his basic wheelchair mobility skills.

\section{CONFLICT OF INTEREST}

The authors declare no conflict of interest.

1 Larsson S, Lepore V, Kennergren C. Atrial myxomas: results of 25 years' experience and review of the literature. Surgery 1989; 105: 695-698.

2 Reynen K. Cardiac myxomas. New Engl J Med 1995; 333: 1610-1617.

3 Shimada Y, Kawata M, Iwasaki Y, Itoh H, Kawachi H. Open heart surgery in a paraplegic patient. Ann Thorac Cardivasc Surg 2004; 10: 304-306.

4 Navi BB, DeAngelis LM. Janeway lesions, Osler nodes, or neither?-Reply. Arch Neurol 2010; 67: 373 . 